\title{
Optimal lower bound for the first eigenvalue of fourth order measure differential equation
}

\author{
Zhou Lijuan $^{1 *}$ (1) and Meng Gang ${ }^{2}$
}

\section{"Correspondence: zlj@amss.ac.cn 'School of Mathematical Sciences, Inner Mongolia University, Huhhot, P.R. China \\ Full list of author information is available at the end of the article}

\begin{abstract}
In this paper, we will find an explicit optimal lower bound for the first eigenvalue of a fourth order measure differential equation, motivated by physical problems when some physically meaningful measurement is fixed. We establish a variational characterization for the first eigenvalue of the fourth order measure differential equation and find the explicit optimal lower bound for the first eigenvalue. As an application, we will find the explicit optimal lower bound for the first eigenvalue of a vibrating beam which represents the smallest axial compressive force necessary to cause the beam to buckle.
\end{abstract}

Keywords: Eigenvalue; Fourth order equation; Minimization problem; Optimal lower bound

\section{Introduction}

Eigenvalue theory has numerous applications in mathematics and applied sciences. Moreover, problems linking the coefficient of an operator to the sequence of its eigenvalues are among the most fascinating of mathematical analysis. The sharp estimate of eigenvalues for ordinary or partial differential equations with different kinds of boundary conditions are very important in the theory of eigenvalues and play very important roles in the study of nonlinear differential equations. For both ordinary and partial differential operators, there have evolved a lot of results $[1,5-7,9,14,18]$.

In this paper, we will study the optimal lower bound for the lowest eigenvalue in the more general setting of measure differential equations (MDEs). Let

$$
\mathcal{M}(I, \mathbb{R}):=\{\mu: I \rightarrow \mathbb{R}: \mu(0+) \exists, \mu(t+)=\mu(t) \forall t \in(0,1), \mathbf{V}(\mu, I)<\infty\}
$$

be the space of non-normalized $\mathbb{R}$-valued measures of $I$. Here $I=[0,1]$ and for any $t \in$ $[0,1), \mu(t+):=\lim _{s \downarrow t} \mu(s)$ is the right limit. The space of (normalized) $\mathbb{R}$-valued measures is

$$
\mathcal{M}_{0}(I, \mathbb{R}):=\{\mu \in \mathcal{M}(I, \mathbb{R}): \mu(0+)=0\} .
$$

(c) The Author(s) 2018. This article is distributed under the terms of the Creative Commons Attribution 4.0 International License (http://creativecommons.org/licenses/by/4.0/), which permits unrestricted use, distribution, and reproduction in any medium, provided you give appropriate credit to the original author(s) and the source, provide a link to the Creative Commons license, and indicate if changes were made. 
We consider the fourth order measure differential equation

$$
\mathrm{d} y^{(3)}(t)+\lambda y^{(2)}(t) \mathrm{d} t+y(t) \mathrm{d} \mu(t)=0, \quad t \in[0,1]
$$

for a given a measure $\mu \in \mathcal{M}_{0}:=\mathcal{M}_{0}(I, \mathbb{R})$ with the asymmetric boundary condition

$$
y(0)=y^{\prime \prime}(0)=0=y(1)=y^{\prime}(1) .
$$

By establishing a variational characterization (3.3) in Sect. 3, we can show existence of an eigenvalue $\lambda=\lambda_{1}(\mu)$. Since the eigenvalue $\lambda$ in (3.3) is characterized by taking minimum over, we call it the first eigenvalue of (1.2)-(1.3). In this paper, we will solve the following minimization problem:

$$
\tilde{\mathbf{L}}(r):=\inf \left\{\lambda_{1}(\mu): \mu \in B_{0}[r]\right\}
$$

here

$$
B_{0}[r]:=\left\{\mu \in \mathcal{M}_{0}(I, \mathbb{R}):\|\mu\|_{\mathbf{V}} \leq r\right\} .
$$

To this end, we will first establish a variational characterization for the first eigenvalue of the fourth order measure differential Eq. (1.2) with boundary (1.3). Then as in [12] for a second order linear MDE, with the strong continuous dependence results of solutions and the zeroth eigenvalues of (1.2) on measures $\mu$ with the weak* topology (see Theorems 2.7 and 3.3), we will find the explicit optimal lower bounds of the first eigenvalue of problem (1.4) as follows.

Theorem 1.1 Given $r \geq 0$, one has

$$
\mathbf{L}(r)=\lambda_{1}\left(-r \delta_{a_{\lambda}}\right) .
$$

Here $\delta_{a_{\lambda}}$ is the Dirac measure at $a_{\lambda}$ and $a_{\lambda}$ satisfies the equation $E(\lambda, a)=0$, where $\delta_{a}$ is as in (4.3) and

$$
\begin{aligned}
E(\lambda, a)= & -4 \sin \omega a-4 \omega a \cos \omega a+2 \omega \cos \omega(2 a-1) \\
& +2 \sin \omega(2 a-1)-2 \omega \cos \omega+4 \omega a \cos \omega+2 \sin \omega .
\end{aligned}
$$

This paper is organized as follows. In Sect. 2, we will recall basic facts on the measure differential equation (MDE). In Sect. 3, we will use the variational method to establish the basic theory for the first eigenvalue of the fourth order MDE. In Sect. 4, based on the minimization characterization of the first eigenvalues we will prove Theorem 1.1. In Sect. 5, As an application of Theorem 1.1, we will find the explicit optimal lower bound for the first eigenvalue of a vibrating beam which represents the smallest axial compressive force necessary to cause the beam to buckle. 


\section{Preliminaries}

In this section, we will recall some basic facts on MDE.

By the Riesz representation theorem $[8],\left(\mathcal{M}_{0}(I, \mathbb{R}),\|\cdot\|_{\mathrm{v}}\right)$ is the same as the dual space of the Banach space $\left(\mathcal{C}(I, \mathbb{R}),\|\cdot\|_{\infty}\right)$ of continuous $\mathbb{R}$-valued functions of $I$ with the supremum norm $\|\cdot\|_{\infty}$, where we write for $\mathbf{V}(m) u I$, as $\|\mu\|_{\mathbf{V}}$ is the total variation of $\mu$ (over $I$ ),

$$
\mathbf{V}(\mu, I):=\sup \left\{\sum_{i=0}^{n-1}\left|\mu\left(t_{i+1}\right)-\mu\left(t_{i}\right)\right|: 0=t_{0}<t_{1}<\cdots<t_{n-1}<t_{n}=1, n \in \mathbb{N}\right\} .
$$

In fact, $\mu \in\left(\mathcal{M}_{0}(I, \mathbb{R}),\|\cdot\|_{\mathrm{v}}\right)$ defines $\mu^{*} \in\left(\mathcal{C}(I, \mathbb{R}),\|\cdot\|_{\infty}\right)^{*}$ by

$$
\mu^{*}(f)=\int_{I} f(t) \mathrm{d} \mu(t), \quad f \in \mathcal{C}(I, \mathbb{R})
$$

which refers to the Riemann-Stieltjes integral, or the Lebesgue-Stieltjes integral [2]. Moreover, one has

$$
\|\mu\|_{\mathbf{V}}=\mathbf{V}(\mu, I)=\sup \left\{\int_{I} f \mathrm{~d} \mu: f \in \mathcal{C}(I, \mathbb{R}),\|f\|_{\infty}=1\right\} .
$$

Lemma 2.1 ([15]) Let $v \in \mathcal{M}_{0}(I, \mathbb{R})$. Define

$$
\hat{v}(t):= \begin{cases}-|v(0)| & \text { for } t=0 \\ \mathbf{V}(v,(0, t]) & \text { for } t \in(0,1]\end{cases}
$$

Then $\hat{v} \in \mathcal{M}_{0}(I, \mathbb{R})$ satisfies $\|v\|_{\mathbf{v}}=\hat{v}(1)-\hat{v}(0)$ and

$$
\left|\int_{[a, b]} f(s) \mathrm{d} v(s)\right| \leq \int_{[a, b]}|f(s)| \mathrm{d} \hat{v}(s) \quad \forall f \in \mathcal{C}(I, \mathbb{R}),[a, b] \subset I .
$$

For the general theory of the Riemann-Stieltjes integral and the Lebesgue-Stieltjes integral, see, e.g., [2].

Let $\ell: I \rightarrow \mathbb{R}$ be $\ell(t) \equiv t$. Then $\ell$ yields the Lebesgue measure of $I$ and the Lebesgue integral. More generally, any $F \in \mathcal{L}^{1}(I, \mathbb{R})$ induces an absolutely continuous measure defined by

$$
\mu_{F}(t):=\int_{[0, t]} F(s) \mathrm{d} s, \quad t \in I .
$$

In this case, one has

$$
\left\|\mu_{F}\right\|_{\mathbf{V}}=\|F\|_{1}=\|F\|_{\mathcal{L}^{1}(I, \mathbb{R})}
$$

and

$$
\int_{I_{0}} f(t) \mathrm{d} \mu_{F}(t)=\int_{I_{0}} f(t) F(t) \mathrm{d} t=\int_{\bar{I}_{0}} f(t) \mathrm{d} \mu_{F}(t)
$$

for any $f \in \mathcal{C}(I, \mathbb{R})$ and subinterval $I_{0} \subset I$. 
In the space $\mathcal{M}_{0}(I, \mathbb{R})$ of measures, one has the usual topology induced by the norm $\|\cdot\|_{\mathbf{V}}$. Due to the duality relation, one has also the following weak* topology $w^{*}$.

Definition 2.2 Let $\mu_{0}, \mu_{n} \in \mathcal{M}_{0}(I, \mathbb{R}), n \in \mathbb{N}$. We say that $\mu_{n}$ is weakly* convergent to $\mu_{0}$ iff, for each $f \in \mathcal{C}(I, \mathbb{R})$, one has

$$
\lim _{n \rightarrow \infty} \int_{I} f \mathrm{~d} \mu_{n}=\int_{I} f \mathrm{~d} \mu_{0}
$$

which refers to the limit of Riemann-Stieltjes integral.

As for the compactness of balls $B_{0}[r]$ in weak* topology, we have the following result.

Lemma $2.3([8])$ Let $r>0$. Then $B_{0}[r] \subset\left(\mathcal{M}_{0}(I, \mathbb{R}), w^{*}\right)$ is sequentially compact.

Definition 2.4 A function $y: I \rightarrow \mathbb{R}$ is called a solution to Eq. (1.2) on the interval $I$ if

- $y \in \mathcal{C}(I, \mathbb{R})$, and

- there exist $\left(y_{0}, y_{1}, y_{2}, y_{3}\right) \in \mathbb{R}^{4}$ and functions $y^{(1)}, y^{(2)}, y^{(3)}:[0,1] \rightarrow \mathbb{R}$ such that the following are satisfied:

$$
\begin{aligned}
& y(t)=y_{0}+\int_{[0, t]} y^{(1)}(s) \mathrm{d} s, \quad t \in[0,1], \\
& y^{(1)}(t)=y_{1}+\int_{[0, t]} y^{(2)}(s) \mathrm{d} s, \quad t \in[0,1], \\
& y^{(2)}(t)=y_{2}+\int_{[0, t]} y^{(3)}(s) \mathrm{d} s, \quad t \in[0,1], \\
& y^{(3)}(t)= \begin{cases}y_{3}, & t=0, \\
y_{3}-\int_{[0, t]} \lambda y^{(2)}(s) \mathrm{d} s-\int_{[0, t]} y(s) \mathrm{d} \mu(s), & t \in(0,1] .\end{cases}
\end{aligned}
$$

The initial condition of MDE (1.2) can be written as

$$
\left(y(0), y^{(1)}(0), y^{(2)}(0), y^{(3)}(0)\right)=\left(y_{0}, y_{1}, y_{2}, y_{3}\right) .
$$

Since we have assumed that $y \in \mathcal{C}:=\mathcal{C}([0,1], \mathbb{R})$, the right-hand sides of $(2.6),(2.7),(2.8)$ are the Lebesgue integrals and (2.9) is the Lebesgue-Stieltjes integral, respectively.

Because solutions of (1.2)-(2.10) are defined via fixed point equations, there are many methods to prove the existence and uniqueness of solutions. For example, one can find a proof from $[4,13,16]$ based on the Kurzweil-Stieltjes integral, which applies also to the first order linear MDE.

Lemma 2.5 For each $\left(y_{0}, y_{1}, y_{2}, y_{3}\right) \in \mathbb{R}^{4}$, problem (1.2)-(2.10) has the unique solution $y(t)$ defined on $[0,1]$.

For $p \in[1, \infty]$, let $\mathcal{L}^{p}:=L^{p}([0,1], \mathbb{R})$ be the Lebesgue space of real-valued functions with the $L^{p}$ norm $\|\cdot\|_{p}$. For $n \in \mathbb{N}$, let $\mathcal{W}^{n, p}:=W^{n, p}([0,1], \mathbb{R})$ and

$$
\mathcal{W}_{0}^{n, p}:=W_{0}^{n, p}([0,1], \mathbb{R})=\left\{y \in \mathcal{W}^{n, p}: y(0)=y(1)=0\right\}
$$


be the usual Sobolev spaces with the norm $\|\cdot\| \mathcal{W}^{n, p}$. For $p=2, \mathcal{W}^{n, 2}$ and $\mathcal{W}_{0}^{n, 2}$ are denoted simply by $\mathcal{H}^{n}$ and $\mathcal{H}_{0}^{n}$, respectively, with the norm $\|\cdot\|_{\mathcal{H}^{n}}$.

By the properties of the Lebesgue integral and the Lebesgue-Stieltjes integral, some regularity results for solutions $y(t)$ are as follows.

Corollary 2.6 Let $y(t)$ be the solution of $(1.2)$. Then $y \in \mathcal{H}^{3}$ and $y^{(3)} \in \mathcal{M}:=\mathcal{M}([0,1], \mathbb{R})$. Hence,

$$
y^{(1)}(t)=y^{\prime}(t) \in \mathcal{C}^{1}:=\mathcal{C}^{1}([0,1], \mathbb{R}), \quad y^{(2)}(t)=y^{\prime \prime}(t) \in \mathcal{A C}:=\mathcal{A C}([0,1], \mathbb{R})
$$

and $y^{(3)}(t)=y^{\prime \prime \prime}(t)$ a.e. $t \in[0,1]$. Here ${ }^{\prime}$ denotes the derivative with respect to $t$.

We use $y\left(t, y_{0}, y_{1}, y_{2}, y_{3}\right)$ to denote the unique solution of (1.2)-(2.10). Let

$$
\begin{array}{ll}
\varphi_{1}(t):=y(t, 1,0,0,0), & \varphi_{2}(t):=y(t, 0,1,0,0), \\
\varphi_{3}(t):=y(t, 0,0,1,0), & \varphi_{4}(t):=y(t, 0,0,0,1),
\end{array}
$$

called the fundamental solutions of (1.2). By the linearity of (1.2) and the uniqueness of the solution, one has, for $t \in[0,1]$,

$$
\left(\begin{array}{c}
y\left(t, y_{0}, y_{1}, y_{2}, y_{3}\right) \\
y^{(1)}\left(t, y_{0}, y_{1}, y_{2}, y_{3}\right) \\
y^{(2)}\left(t, y_{0}, y_{1}, y_{2}, y_{3}\right) \\
y^{(3)}\left(t, y_{0}, y_{1}, y_{2}, y_{3}\right)
\end{array}\right)=\left(\begin{array}{cccc}
\varphi_{1}(t) & \varphi_{2}(t) & \varphi_{3}(t) & \varphi_{4}(t) \\
\varphi_{1}^{(1)}(t) & \varphi_{2}^{(1)}(t) & \varphi_{3}^{(1)}(t) & \varphi_{4}^{(1)}(t) \\
\varphi_{1}^{(2)}(t) & \varphi_{2}^{(2)}(t) & \varphi_{3}^{(2)}(t) & \varphi_{4}^{(2)}(t) \\
\varphi_{1}^{(3)}(t) & \varphi_{2}^{(3)}(t) & \varphi_{3}^{(3)}(t) & \varphi_{4}^{(3)}(t)
\end{array}\right)\left(\begin{array}{c}
y_{0} \\
y_{1} \\
y_{2} \\
y_{3}
\end{array}\right)
$$

In [12], the authors have obtained the continuity of solutions in measures for the second order linear MDE. For the fourth order linear MDE, we can argue in a similar way to prove the following conclusion.

Theorem 2.7 Let $y(t, \mu)$ be the solution of (1.2)-(2.10). Then the following solution mappings are continuous:

$$
\begin{array}{rlrl}
\left(\mathcal{M}_{0}, w^{*}\right) & \rightarrow\left(\mathcal{C},\|\cdot\|_{\infty}\right), & \mu & \rightarrow y(\cdot, \mu), \\
\left(\mathcal{M}_{0}, w^{*}\right) \rightarrow\left(\mathcal{C},\|\cdot\|_{\infty}\right), & \mu & \rightarrow y^{(1)}(\cdot, \mu), \\
\left(\mathcal{M}_{0}, w^{*}\right) \rightarrow\left(\mathcal{C},\|\cdot\|_{\infty}\right), & \mu \rightarrow y^{(2)}(\cdot, \mu), \\
\left(\mathcal{M}_{0}, w^{*}\right) \rightarrow\left(\mathcal{M}, w^{*}\right), & \mu & \rightarrow y^{(3)}(\cdot, \mu) .
\end{array}
$$

By Corollary 2.6, we have the following corollary.

\section{Corollary 2.8 The solution mapping}

$$
\left(\mathcal{M}_{0}, w^{*}\right) \rightarrow\left(\mathcal{C}^{2},\|\cdot\|_{\mathcal{H}^{2}}\right), \quad \mu \rightarrow y(\cdot, \mu)
$$

is continuous, where $\mathcal{C}^{2}:=\mathcal{C}^{2}([0,1], \mathbb{R})$. 


\section{Variational characterization for the first eigenvalue of MDE}

In this section, we will use the variational method to establish the basic theory for the first eigenvalue of the fourth order MDE. Though the question what is the complete structure of eigenvalues of problem (1.2)-(1.3) remains open to us, it is a standard result that any (possible) eigenvalue $\lambda \in \mathbb{R}$ of problem (1.2)-(1.3) with eigenfunction $u \in \mathcal{H}_{0 x 0 x}^{3}$ must satisfy

$$
\lambda=\frac{\int_{[0,1]}\left(u^{\prime \prime}\right)^{2} \mathrm{~d} t+\int_{[0,1]} u^{2} \mathrm{~d} \mu(t)}{\int_{[0,1]}\left(u^{\prime}\right)^{2} \mathrm{~d} t},
$$

where

$$
\mathcal{H}_{0 x 0 x}^{3}:=\left\{y \in \mathcal{H}^{3}: y(0)=y^{\prime \prime}(0)=y(1)=y^{\prime}(1)=0\right\} .
$$

In the following theorem, we show that problem (1.2)-(1.3) does admit the first eigenvalue and we explicitly present the minimization characterizations as follows.

Theorem 3.1 Let $\mu \in \mathcal{M}_{0}$, problem (1.2)-(1.3) admits the lowest eigenvalue $\lambda_{1}(\mu)$, which has the following minimization characterizations:

$$
\lambda_{1}(\mu)=\min _{u \in \mathcal{H}_{0 x 0 x}^{3} \backslash\{0\}} \frac{\int_{[0,1]}\left(u^{\prime \prime}\right)^{2} \mathrm{~d} t+\int_{[0,1]} u^{2} \mathrm{~d} \mu(t)}{\int_{[0,1]}\left(u^{\prime}\right)^{2} \mathrm{~d} t} .
$$

Here $\mathcal{M}_{0}$ is as in (1.1).

Proof Denote

$$
R(u):=\frac{\int_{[0,1]}\left(u^{\prime \prime}\right)^{2} \mathrm{~d} t+\int_{[0,1]} u^{2} \mathrm{~d} \mu(t)}{\int_{[0,1]}\left(u^{\prime}\right)^{2} \mathrm{~d} t}
$$

where

$$
u \in \mathcal{H}_{0 x}^{2}:=\left\{y \in \mathcal{H}^{2}: y(0)=y(1)=y^{\prime}(1)=0\right\}
$$

With the standard estimate we have

$$
\begin{aligned}
\|u\|_{\infty}^{2} & \leq\left(\int_{[0,1]}\left|u^{\prime}\right| \mathrm{d} t\right)^{2} \leq \int_{[0,1]} u^{\prime} u^{\prime} \mathrm{d} t \\
& =\left.u u^{\prime}\right|_{0} ^{1}-\int_{[0,1]} u u^{\prime \prime} \mathrm{d} t \leq \int_{[0,1]}\left|u u^{\prime \prime}\right| \mathrm{d} t \\
& \leq\|u\|_{2}\left\|u^{\prime \prime}\right\|_{2},
\end{aligned}
$$

i.e.,

$$
\left\|u^{\prime \prime}\right\|_{2} \geq\|u\|_{\infty}^{2} /\|u\|_{2} .
$$


Since

$$
\begin{aligned}
\int_{[0,1]} u^{2} \mathrm{~d} \mu(t) & \geq-\|\mu\|_{\mathbf{V}}\|u\|_{\infty}^{2}=-\frac{\|\mu\|_{\mathbf{V}}\|u\|_{2}}{\sqrt{2}} \cdot \frac{\sqrt{2}\|u\|_{\infty}^{2}}{\|u\|_{2}} \\
& \geq-\frac{1}{2}\left(\frac{\|\mu\|_{\mathbf{V}}^{2}\|u\|_{2}^{2}}{2}+\frac{2\|u\|_{\infty}^{4}}{\|u\|_{2}^{2}}\right)
\end{aligned}
$$

Thus, we have from (3.6) and (3.7)

$$
R(u) \geq-\frac{\|\mu\|_{\mathbf{V}}^{2}}{4} \quad \forall 0 \neq u \in \mathcal{H}_{0 x}^{2} .
$$

Due to (3.8), one has

$$
\lambda_{1}:=\inf _{u \in \mathcal{H}_{0 x}^{2} \backslash\{0\}} R(u)>-\infty .
$$

Take a sequence $\left\{u_{n}\right\} \subset \mathcal{H}_{0 x}^{2}$ such that

$$
\left\|u_{n}^{\prime}\right\|_{\infty}=1 \text { and } \lim _{n \rightarrow+\infty} R\left(u_{n}\right)=\lambda_{1} \text {. }
$$

Then

$$
\int_{[0,1]}\left(u_{n}^{\prime \prime}\right)^{2} \mathrm{~d} t=R\left(u_{n}\right) \int_{[0,1]} u_{n}^{\prime 2} \mathrm{~d} t-\int_{[0,1]} u_{n}^{2} \mathrm{~d} \mu(t) \leq\left|R\left(u_{n}\right)\right|+\|\mu\|_{\mathbf{V}}
$$

is bounded and $\left\{u_{n}\right\} \subset \mathcal{H}_{0 x}^{2}$ is bounded. As $\mathcal{H}_{0 x}^{2}$ is a Hilbert space and is compactly embedded into $\mathcal{C}^{1}$, there exists a non-zero $u_{0} \in \mathcal{H}_{0 x}^{2}$ such that

$$
u_{n} \rightarrow u_{0} \quad \text { in }\left(\mathcal{H}_{0 x}^{2}, w\right) \quad \text { and } \quad u_{n} \rightarrow u_{0} \quad \text { in }\left(\mathcal{C}^{1},\|\cdot\|_{\mathcal{C}^{1}}\right)
$$

going to a subsequence if necessary. Thus

$$
\int_{[0,1]}\left(u_{0}^{\prime \prime}\right)^{2} \mathrm{~d} t=\lim _{n \rightarrow+\infty} \int_{[0,1]} u_{0}^{\prime \prime} u_{n}^{\prime \prime} \mathrm{d} t \leq \liminf _{n \rightarrow+\infty}\left(\int_{[0,1]}\left(u_{0}^{\prime \prime}\right)^{2} \mathrm{~d} t\right)^{1 / 2}\left(\int_{[0,1]}\left(u_{n}^{\prime \prime}\right)^{2} \mathrm{~d} t\right)^{1 / 2}
$$

This implies that

$$
\begin{aligned}
R\left(u_{0}\right) & \leq \frac{\liminf _{n \rightarrow+\infty} \int_{[0,1]}\left(u_{n}^{\prime \prime}\right)^{2} \mathrm{~d} t+\int_{[0,1]} u_{0}^{2} \mathrm{~d} \mu(t)}{\int_{[0,1]} u_{0}^{\prime 2} \mathrm{~d} t} \\
& =\liminf _{n \rightarrow+\infty} \frac{\int_{[0,1]}\left(u_{n}^{\prime \prime}\right)^{2} \mathrm{~d} t+\int_{[0,1]} u_{n}^{2} \mathrm{~d} \mu(t)}{\int_{[0,1]} u_{n}^{\prime 2} \mathrm{~d} t} \\
& =\liminf _{n \rightarrow+\infty} R\left(u_{n}\right)=\lambda_{1} .
\end{aligned}
$$

Combining with (3.9), one has

$$
R\left(u_{0}\right)=\lambda_{1}=\min _{u \in \mathcal{H}_{0 x}^{2} \backslash\{0\}} R(u) .
$$


Take any

$$
\phi \in \mathcal{C}_{c}^{\infty}:=\left\{\phi \in C^{\infty}([0,1]): \operatorname{supp} \phi \subset(0,1)\right\}
$$

Then $u_{0}+s \phi \in \mathcal{H}_{0 x}^{2} \backslash\{0\}$ for all $s \in \mathbb{R}$ with $|s|$ small enough.

As a function of $s$, it follows from (3.11) that $R\left(u_{0}+s \phi\right)$ takes a minimum at $s=0$.

Thus

$$
\begin{aligned}
0= & \left.\frac{\mathrm{d} R\left(u_{0}+s \phi\right)}{\mathrm{d} s}\right|_{s=0} \\
= & \frac{2}{\left(\int_{[0,1]} u_{0}^{\prime 2} \mathrm{~d} t\right)^{2}}\left(\int_{[0,1]} u_{0}^{\prime 2} \mathrm{~d} t\left(\int_{[0,1]} u_{0}^{\prime \prime} \phi^{\prime \prime} \mathrm{d} t+\int_{[0,1]} u_{0} \phi \mathrm{d} \mu(t)\right)\right. \\
& \left.-\left(\int_{[0,1]}\left(u_{0}^{\prime \prime}\right)^{2} \mathrm{~d} t+\int_{[0,1]} u_{0}^{2} \mathrm{~d} \mu(t)\right) \int_{[0,1]} u_{0}^{\prime} \phi^{\prime} \mathrm{d} t\right) \\
= & \frac{2}{\int_{[0,1]} u_{0}^{2} \mathrm{~d} t}\left(\int_{[0,1]} u_{0}^{\prime \prime} \phi^{\prime \prime} \mathrm{d} t+\int_{[0,1]} u_{0} \phi \mathrm{d} \mu(t)-\lambda_{1} \int_{[0,1]} u_{0}^{\prime} \phi^{\prime} \mathrm{d} t\right) .
\end{aligned}
$$

Here (3.11) is used and the derivative is found using definition (3.4) for $R(u)$.

Since $\phi \in \mathcal{C}_{c}^{\infty}$, one has

$$
\phi^{\prime}(t)=\int_{[0, t]} \phi^{\prime \prime}(s) \mathrm{d} s
$$

and

$$
\phi(t)=\int_{[0, t]}\left(\int_{[0, s]} \phi^{\prime \prime}(\tau) \mathrm{d} \tau\right) \mathrm{d} s=\int_{[0, t]}(t-s) \phi^{\prime \prime}(s) \mathrm{d} s .
$$

Then

$$
\begin{aligned}
\int_{[0,1]} u_{0}^{\prime}(t) \phi^{\prime}(t) \mathrm{d} t & =\int_{[0,1]}\left(\int_{[0, t]} u_{0}^{\prime}(t) \phi^{\prime \prime}(s) \mathrm{d} s\right) \mathrm{d} t \\
& =\int_{[0,1]}\left(\int_{(s, 1]} u_{0}^{\prime}(t) \mathrm{d} t\right) \phi^{\prime \prime}(s) \mathrm{d} s \\
& =\int_{[0,1]}\left(\int_{(t, 1]} u_{0}^{\prime}(s) \mathrm{d} s\right) \phi^{\prime \prime}(t) \mathrm{d} t
\end{aligned}
$$

and

$$
\begin{aligned}
\int_{[0,1]} u_{0}(t) \phi(t) \mathrm{d} \mu(t) & =\int_{[0,1]}\left(\int_{[0, t]}(t-s) u_{0}(t) \phi^{\prime \prime}(s) \mathrm{d} s\right) \mathrm{d} \mu(t) \\
& =\int_{[0,1]}\left(\int_{(s, 1]}(t-s) u_{0}(t) \mathrm{d} \mu(t)\right) \phi^{\prime \prime}(s) \mathrm{d} s \\
& =\int_{[0,1]}\left(\int_{(t, 1]}(s-t) u_{0}(s) \mathrm{d} \mu(s)\right) \phi^{\prime \prime}(t) \mathrm{d} t .
\end{aligned}
$$

Substituting into (3.12), we obtain

$$
\int_{[0,1]}\left(u_{0}^{\prime \prime}(t)+\int_{(t, 1]}(s-t) u_{0}(s) \mathrm{d} \mu(s)-\lambda_{1} \int_{(t, 1]} u_{0}^{\prime}(s) \mathrm{d} s\right) \phi^{\prime \prime}(t) \mathrm{d} t=0
$$


for all $\phi \in \mathcal{C}_{c}^{\infty}$. Hence $u_{0}(t)$ satisfies

$$
u_{0}^{\prime \prime}(t)+\int_{(t, 1]}(s-t) u_{0}(s) \mathrm{d} \mu(s)-\lambda_{1} \int_{(t, 1]} u_{0}^{\prime}(s) \mathrm{d} s=c t+\hat{c} \quad \text { a.e. } t \in[0,1]
$$

where $c$ and $\hat{c}$ are constants. Note that

$$
\begin{aligned}
\int_{[0, t]}\left(\int_{[0, \tau]} u_{0}(s) \mathrm{d} \mu(s)\right) \mathrm{d} \tau & =\int_{[0, t]}(t-s) u_{0}(s) \mathrm{d} \mu(s) \\
& =\int_{(t, 1]}(s-t) u_{0}(s) \mathrm{d} \mu(s)+\int_{[0,1]}(t-s) u_{0}(s) \mathrm{d} \mu(s) \\
& =\int_{(t, 1]}(s-t) u_{0}(s) \mathrm{d} \mu(s)+c_{1} t+\hat{c}_{1},
\end{aligned}
$$

where $c_{1}$ and $\hat{c}_{1}$ are constants. Hence Eq. (3.13) can be rewritten as

$$
u_{0}^{\prime \prime}(t)+\int_{[0, t]}\left(\int_{[0, \tau]} u_{0}(s) \mathrm{d} \mu(s)\right) \mathrm{d} \tau-\lambda_{1} \int_{(t, 1]} u_{0}^{\prime}(s) \mathrm{d} s=c_{2} t+\hat{c}_{2} \quad \text { a.e. } t \in[0,1]
$$

where $c_{2}=c-c_{1}$ and $\hat{c}_{2}=\hat{c}-\hat{c}_{1}$ By the properties of Lebesgue integral and LebesgueStieltjes integral, one knows from (3.14) that $u_{0}^{\prime \prime}(t)$ is absolutely continuous and satisfies

$$
u_{0}^{\prime \prime \prime}(t)+\int_{[0, t]} u_{0}(s) \mathrm{d} \mu(s)+\lambda_{1} \int_{[0, t]} u_{0}^{\prime \prime}(s) \mathrm{d} s=\tilde{c} \quad \text { a.e. } t \in[0,1]
$$

Equation (3.15) implies that $u_{0} \in \mathcal{H}_{0 x 0 x}^{3}$ and $\lambda_{1}$ is necessarily an eigenvalue of problem (1.2) -(1.3) with the eigenfunction $u_{0}$. Because of (3.11) and the fact that $u_{0} \in \mathcal{H}_{0 x 0 x}^{3}, \lambda_{1}=$ $\lambda_{1}(\mu)$, which is characterized as in (3.3) and we know that $\lambda_{1}(\mu)$ must be the smallest eigenvalue of problem (1.2)-(1.3).

Let us introduce the following ordering for measures. We say that measures $\mu_{2} \geq \mu_{1}$ if

$$
\int_{[0,1]} f(t) \mathrm{d} \mu_{2}(t) \geq \int_{[0,1]} f(t) \mathrm{d} \mu_{1}(t) \quad \text { for all } f \in \mathcal{C}_{+}:=\{f \in \mathcal{C}: f(t) \geq 0, t \in[0,1]\}
$$

As a consequence of (3.3) in Theorem 3.1, we can obtain the following result.

\section{Corollary 3.2 One has}

$$
\mu_{2} \geq \mu_{1} \quad \Rightarrow \quad \lambda_{1}\left(\mu_{2}\right) \geq \lambda_{1}\left(\mu_{1}\right)
$$

for $\mu_{1}, \mu_{2} \in \mathcal{M}_{0}$.

Now the continuity of the first eigenvalue in measures with the weak* topology can be proved by the same arguments as those in [10].

Theorem $3.3 \lambda_{1}(\mu)$ is continuous in $\mu$ with weak ${ }^{*}$ topology in $\mathcal{M}_{0}$. 


\section{The optimal lower bound of the first eigenvalue of MDE}

In this section, we will solve the minimization problem (1.4) explicitly for the lowest eigenvalue $\lambda_{1}$. To this end, we use the lemma that follows.

Lemma 4.1 Given $r>0$, consider the following minimization problem:

$$
\tilde{\mathbf{L}}(r):=\inf \left\{\lambda_{1}(\mu): \mu \in B_{0}[r]\right\}=\min \left\{\lambda_{1}(\mu): \mu \in B_{0}[r]\right\} .
$$

One has

$$
\tilde{\mathbf{L}}(r)=\inf _{a \in(0,1)} \lambda_{1}\left(-r \delta_{a}\right)
$$

where for $a \in(0,1]$

$$
\delta_{a}(t)= \begin{cases}0 & \text { for } t \in[0, a), \\ 1 & \text { for } t \in[a, 1],\end{cases}
$$

is the unit Dirac measure at $t=a$.

Proof Given $\mu \in B_{0}[r]$, we take an eigenfunction $y(t)$ associated with $\lambda_{1}(\mu)$ which satisfies the normalization condition $\left\|y^{\prime}\right\|_{2}=1$. There exists $a \in(0,1)$ such that

$$
\|y\|_{\infty}=\max _{t \in[0,1]}|y(t)|=|y(a)| .
$$

We have

$$
\begin{aligned}
\lambda_{1}(\mu) & =\int_{[0,1]}\left(y^{\prime \prime}\right)^{2} \mathrm{~d} t+\int_{[0,1]} y^{2} \mathrm{~d} \mu(t) \\
& \geq \int_{[0,1]}\left(y^{\prime \prime}\right)^{2} \mathrm{~d} t-\|\mu\|_{\mathbf{V}}\|y\|_{\infty}^{2} \\
& \geq \int_{[0,1]}\left(y^{\prime \prime}\right)^{2} \mathrm{~d} t-r y^{2}(a) \\
& =\int_{[0,1]}\left(y^{\prime \prime}\right)^{2} \mathrm{~d} t+\int_{[0,1]} y^{2} \mathrm{~d}\left(-r \delta_{a}(t)\right) \\
& \geq \lambda_{1}\left(-r \delta_{a}\right) .
\end{aligned}
$$

Here the last inequality in (4.4) follows from characterization (3.3) for $\lambda_{1}\left(-r \delta_{a}\right)$ since $\left\|y^{\prime}\right\|_{2}=1$. Hence

$$
\tilde{\mathbf{L}}(r)=\inf _{a \in(0,1)} \lambda_{1}\left(-r \delta_{a}\right) .
$$

The proof of Theorem 1.1 Let us explicitly find the first eigenvalue for Dirac measures $-r \delta_{a}$, where $a \in(0,1)$ and $r \geq 0$. To this end, we need to solve the following equation:

$$
\mathrm{d} y^{(3)}(t)+\lambda y^{(2)}(t) \mathrm{d} t-r y(t) \mathrm{d} \delta_{a}(t)=0, \quad t \in[0,1] .
$$


One knows that a solution $y(t)$ of (4.5) satisfies the classical ODE

$$
y^{\prime \prime \prime \prime}(t)+\lambda y^{\prime \prime}(t)=0
$$

for $t$ on the intervals $[0, a)$ and $(a, 1]$. At $t=a$, one has the following relations:

$$
\begin{cases}y(a+)=y(a-), & y^{\prime}(a+)=y^{\prime}(a-), \\ y^{\prime \prime}(a+)=y^{\prime \prime}(a-), & y^{\prime \prime \prime}(a+)=y^{\prime \prime \prime}(a-)+r y(a-) .\end{cases}
$$

From the first two conditions of (1.3), let us consider the initial value

$$
\left(y(0), y^{\prime}(0), y^{\prime \prime}(0), y^{\prime \prime \prime}(0)\right)=(0, c, 0, \hat{c}) \neq 0,
$$

and we obtain

$$
y(t)=c t+\hat{c}\left(\frac{1}{\omega^{2}} t+\frac{-1}{\omega^{3}} \sin \omega t\right)=c_{1} t+c_{2} \sin \omega t
$$

for $t \in[0, a)$ and $\left(c_{1}, c_{2}\right) \neq 0$. Here,

$$
\omega:= \begin{cases}\sqrt[2]{\lambda} \in \mathbb{R} & \text { for } \lambda \geq 0 \\ \sqrt[2]{|\lambda|} i \in \mathbb{C} & \text { for } \lambda<0\end{cases}
$$

By (4.7), we have

$$
\begin{array}{ll}
y(a+)=c_{1} a+c_{2} \sin \omega a, & y^{\prime}(a+)=c_{1}+c_{2} \omega \cos \omega a, \\
y^{\prime \prime}(a+)=-c_{2} \omega^{2} \sin \omega a, & y^{\prime \prime \prime}(a+)=-c_{2} \omega^{3} \cos \omega a+r\left(c_{1} a+c_{2} \sin \omega a\right) .
\end{array}
$$

By using this as the initial value at $t=a$, we obtain from ODE (4.6)

$$
y(t)=c_{1} t+c_{2} \sin \omega t+\left(r\left(c_{1} a+c_{2} \sin \omega a\right)\right)\left(\frac{1}{\omega^{2}}(t-a)+\frac{-1}{\omega^{3}} \sin \omega(t-a)\right)
$$

and

$$
y^{\prime}(t)=c_{1}+c_{2} \omega \cos \omega t+\left(r\left(c_{1} a+c_{2} \sin \omega a\right)\right)\left(\frac{1}{\omega^{2}}+\frac{-1}{\omega^{2}} \cos \omega(t-a)\right)
$$

for $t \in(a, 1]$. Now the last two conditions $y(1)=y^{\prime}(1)=0$ of (1.3) imply the following linear system for $\left(c_{1}, c_{2}\right)$ :

$$
\left\{\begin{array}{l}
c_{1}+c_{2} \sin \omega+r\left(c_{1} a+c_{2} \sin \omega a\right)\left(\frac{1}{\omega^{2}}(1-a)+\frac{-1}{\omega^{3}} \sin \omega(1-a)\right)=0 \\
c_{1}+c_{2} \omega \cos \omega+\left(r\left(c_{1} a+c_{2} \sin \omega a\right)\right)\left(\frac{1}{\omega^{2}}+\frac{-1}{\omega^{2}} \cos \omega(1-a)\right)=0
\end{array}\right.
$$

In order that system (4.11) has non-zero solutions $\left(c_{1}, c_{2}\right)$, the corresponding determinant of (4.11) is necessarily zero. This yields the following equation:

$$
G(\lambda, a)=r,
$$


where $G:\left(-\infty, \pi_{0}^{2}\right] \times(0,1) \rightarrow[0,+\infty)$ is defined as

$$
G(\lambda, a):= \begin{cases}\frac{2 \omega^{3}(-\sin \omega+\omega \cos \omega)}{\tilde{G}(\omega, a)} & \text { for } \lambda \neq 0, \\ \frac{12}{a^{2}(a+3)(1-a)^{3}} & \text { for } \lambda=0,\end{cases}
$$

where

$$
\begin{aligned}
\widetilde{G}(\omega, a):= & -4 \omega a \sin \omega a+\omega \sin (2 \omega a-\omega)+\cos \omega-\cos (2 \omega a-\omega) \\
& -2 \omega^{2} a \cos \omega+\omega \sin \omega+2 \omega^{2} a^{2} \cos \omega+2 \omega a \sin \omega,
\end{aligned}
$$

$\pi_{0}(\approx 4.4934)$ is the minimal positive root of the equation $x \cos x-\sin x=0$. Then, by the existence of the first eigenvalue, we conclude

$$
\lambda_{1}\left(-r \delta_{a}\right)=\min \{\lambda \in \mathbb{R}: G(\lambda, a)-r=0\} .
$$

It is easy to check that $G(\lambda, a)$ is a well-defined real function of $(\lambda, a) \in\left(-\infty, \pi_{0}^{2}\right] \times(0,1)$ with $G\left(\pi_{0}^{2}, a\right)=0$ and $G(\lambda, a)=G(\lambda, 1-a)$. Moreover, the following properties of $G(\lambda, a)$ can be proved with a standard calculation.

(P1) When $a \in(0,1)$ is fixed, $G(\lambda, a)$ is decreasing in $\lambda \in\left(-\infty, \pi_{0}^{2}\right]$;

(P2) when $\lambda \in\left(-\infty, \pi_{0}^{2}\right]$ is fixed, $G(\lambda, a)$ is decreasing in $a \in\left(0, a_{\lambda}\right)$, where $a_{\lambda} \in(0,1)$ is such that

$$
G\left(\lambda, a_{\lambda}\right)=\min _{a \in(0,1)} G(\lambda, a)
$$

and $E\left(\lambda, a_{\lambda}\right)=0$, where $E:\left(-\infty, \pi_{0}^{2}\right] \times(0,1) \rightarrow \mathbb{R}$ is defined as in (1.6).

Since

$$
\lim _{a \rightarrow 0} G(\lambda, a)=\lim _{a \rightarrow 1} G(\lambda, a)=+\infty
$$

there exists $a_{\lambda} \in(0,1)$ such that

$$
G\left(\lambda, a_{\lambda}\right)=\min _{a \in(0,1)} G(\lambda, a)
$$

which is equivalent to

$$
\widetilde{G}\left(\omega, a_{\lambda}\right)=\max _{a \in(0,1)} \widetilde{G}(\omega, a) .
$$

Thus one has $0=\left.\frac{\partial}{\partial a} \widetilde{G}(\omega, a)\right|_{a=a_{\lambda}}$ and $\left.\frac{\partial^{2}}{\partial a^{2}} \widetilde{G}(\omega, a)\right|_{a=a_{\lambda}}>0$ which implies $E\left(\lambda, a_{\lambda}\right)=0$.

By properties (P1) and (P2), we have

$$
\lambda_{1}\left(-r \delta_{a_{\lambda}}\right)=\inf _{a \in(0,1)} \lambda_{1}\left(-r \delta_{a}\right)
$$

with which one has

$$
\tilde{\mathbf{L}}(r)=\lambda_{1}\left(-r \delta_{a_{\lambda}}\right) .
$$


Remark 4.2 In $[11,17]$, the authors have solved the minimization problem of eigenvalues of the second order equation and pointed out that the critical measure of the Dirichlet eigenvalues is located at the center $a=\frac{1}{2}$. By the conclusion of Theorem 1.1 one can check that $a_{\lambda} \neq \frac{1}{2}$ with a straightforward calculation, which illustrates the very interesting phenomenon that the minimizing measure will no longer be located at the center of the interval (i.e. $a=\frac{1}{2}$ ) when the boundary condition is asymmetric.

\section{Application to ODE}

The eigenvalue problem of a vibrating beam described by the fourth order ODE

$$
y^{\prime \prime \prime \prime}(t)+\lambda y^{\prime \prime}(t)+F(t) y(t)=0, \quad t \in[0,1]
$$

with the boundary condition (1.3) describes a vibrating beam which is subject to an axial compressive load $\lambda$ that causes it to buckle. It is well known that (5.1)-(1.3) admits a sequence of eigenvalues $\lambda_{1}(F)<\lambda_{2}(F)<\cdots<\lambda_{m}(F)<\cdots$. Associated with each eigenvalue, there is only one linear independent eigenfunction (see [3]). Specifically, $\lambda_{1}(F)$ is called the first eigenvalue and represents the smallest axial compressive load. People are concerned with the lowest eigenvalues $\lambda_{1}(F)$ which represents the smallest axial compressive force necessary to cause the beam to buckle. To get their sharp lower bounds one has to solve the following minimization problem:

$$
\mathbf{L}(r):=\inf \left\{\lambda_{1}(F): F \in B_{1}[r]\right\}
$$

Here $B_{1}[r]:=\left\{F \in \mathcal{L}^{1}:\|F\|_{1} \leq r\right\}, r>0$.

We usually do not know if minimization problem (5.2) can be attained by some $F$ from $B_{1}[r]$ since the $L^{1}$ balls $B_{1}[r]$ lack compactness even in the weak topology of $\mathcal{L}^{1}$. Precisely, these extremal value problems cannot be solved directly by variational methods, because eigenvalues $\lambda_{n}(F)$ are implicit functionals of potentials $F$, the space $\mathcal{L}^{1}$ is infinite dimensional, and the balls $B_{1}[r]$ with radius $r$ in $\mathcal{L}^{1}$ are non-compact non-smooth sets.

As an application of results of Theorem 1.1, we can overcome the difficulties described above by building a relationship between the minimization problem of the first eigenvalue for the ODE and the one for the MDE. With the conclusion we obtained for MDE we give the explicit optimal lower bound for the first eigenvalue of the vibrating beam (5.1) with boundary condition (1.3).

In general, a measure cannot be a limit of smooth measures in the norm $\|\cdot\|_{\mathrm{v}}$. However, in the $w^{*}$ topology, the following conclusion holds.

Lemma 5.1 ([9]) Given $\mu_{0} \in \mathcal{M}_{0}(I, \mathbb{R})$, there exists a sequence of measures $\left\{\mu_{n}\right\} \subset$ $C^{\infty}(I, \mathbb{R}) \cap \mathcal{M}_{0}(I, \mathbb{R})$ such that

$$
\mu_{n} \rightarrow \mu_{0} \quad \operatorname{in}\left(\mathcal{M}_{0}(I, \mathbb{R}), w^{*}\right) .
$$

Moreover, if $\mu_{0}$ is increasing (decreasing) on $I$, then the sequence $\left\{\mu_{n}\right\}$ above can be chosen such that, for each $n \in \mathbb{N}, \mu_{n}$ is increasing (deceasing) on I and $\left\|\mu_{n}\right\|_{\mathbf{V}}=\left\|\mu_{0}\right\|_{\mathbf{V}}$.

Finally, because of Lemma 5.1 and Theorem 3.3, we can obtain the relationship between the minimization problems of ODE and of MDE as follows. 
Theorem 5.2 Given $r \geq 0$, one has that

$$
\mathbf{L}(r)=\tilde{\mathbf{L}}(r) .
$$

Proof Given $F \in B_{1}[r]$, the measure $\mu_{F} \in \mathcal{M}_{0}$ is defined as

$$
\mu_{F}(t):=\int_{[0, t]} F(s) \mathrm{d} s, \quad t \in I
$$

and we see that $\mu_{F} \in B_{0}[r]$ is absolutely continuous with respect to the Lebesgue measure.

So for any $F \in B_{1}[r]$,

$$
\tilde{\mathbf{L}}(r) \leq \lambda_{1}\left(\mu_{F}\right)=\lambda_{1}(F)
$$

which implies that

$$
\tilde{\mathbf{L}}(r) \leq \mathbf{L}(r)
$$

On the other hand, there exists $\bar{\mu} \in B_{0}[r]$ such that $\lambda_{1}(\bar{\mu})=\tilde{\mathbf{L}}(r)$. By the monotonicity of $\lambda_{1}(\mu)$, without loss of generality, we can assume that $\bar{\mu}=-\hat{\bar{\mu}}$ is decreasing. By Lemma 5.1, there exists a sequence of measures $\left\{\bar{\mu}_{n}\right\} \subset C^{\infty} \cap \mathcal{M}_{0}$ such that

$$
\begin{aligned}
& \frac{\mathrm{d} \bar{\mu}_{n}(t)}{\mathrm{d} t}=\bar{F}_{n}(t), \\
& \left\|\bar{\mu}_{n}\right\|_{\mathbf{V}}=\left\|\bar{F}_{n}\right\|_{1}=\|\bar{\mu}\|_{\mathbf{V}} \leq r, \\
& \bar{\mu}_{n} \rightarrow \bar{\mu} \quad \text { in }\left(\mathcal{M}_{0}, w^{*}\right) .
\end{aligned}
$$

Therefore, by Theorem 3.3, we have

$$
\tilde{\mathbf{L}}(r)=\lambda_{1}(\bar{\mu})=\lim _{n \rightarrow \infty} \lambda_{1}\left(\bar{\mu}_{n}\right)=\lim _{n \rightarrow \infty} \lambda_{1}\left(\bar{F}_{n}\right) \geq \lim _{n \rightarrow \infty} \mathbf{L}(r)=\mathbf{L}(r) .
$$

Now (5.5) and (5.6) imply that $\mathbf{L}(r)=\tilde{\mathbf{L}}(r)$.

Remark 5.3 To compute $\mathbf{L}(r)$, it suffices to solve the following optimization problem:

$$
\min f(\lambda, a)=\lambda
$$

subject to the constraints

$$
G(\lambda, a)-r=0, \quad 0<a<a_{\lambda}
$$

where $G(\lambda, a)$ as in (4.13). 


\section{Funding}

The first author is supported by the scientific starting research foundation of Inner Mongolia University (No.

20100-5165106). The second author is supported by the National Natural Science Foundation of China (Grant No. 11671378) .

\section{Availability of data and materials}

Data sharing not applicable to this article as no datasets were generated or analyzed during the current study.

\section{Competing interests}

The authors declare that they have no competing interests.

\section{Authors' contributions}

Both authors have equally contributed to this article and read and approved the final manuscript.

\section{Author details}

${ }^{1}$ School of Mathematical Sciences, Inner Mongolia University, Huhhot, P.R. China. ${ }^{2}$ School of Mathematical Sciences, University of Chinese Academy of Sciences, Beijing, P.R. China.

\section{Publisher's Note}

Springer Nature remains neutral with regard to jurisdictional claims in published maps and institutional affiliations.

Received: 15 August 2018 Accepted: 4 December 2018 Published online: 12 December 2018

\section{References}

1. Banks, D.O.: Bounds for the eigenvalues of nonhomogeneous hinged vibrating rod. J. Math. Mech. 16, 949-966 (1967)

2. Carter, M., van Brunt, B.: The Lebesque-Stieltjes Integral: A Practical Introduction. Springer, New York (2000)

3. Courant, R., Hilbert, D.: Methods of Mathematical Physics. Wiley, New York (1953)

4. Halas, Z., Tvrdý, M.: Continuous dependence of solutions of generalized linear differential equations on a parameter. Funct. Differ. Equ. 16, 299-313 (2009)

5. Kao, C.-Y., Lou, Y., Yanagida, E.: Principal eigenvalue for an elliptic problem with indefinite weight on cylindrical domains. Math. Biosci. Eng. 5, 315-335 (2008)

6. Karaa, S.: Sharp estimates for the eigenvalues of some differential equations. SIAM J. Math. Anal. 29, 1279-1300 (1998)

7. Krein, M.G.: On certain problems on the maximum and minimum of characteristic values and on the Lyapunov zones of stability. Am. Math. Soc. Transl. Ser. 2 1, 163-187 (1955)

8. Megginson, R.E.: An Introduction to Banach Space Theory. Graduate Texts in Mathematics, vol. 183. Springer, New York (1998)

9. Meng, G.: Extremal problems for eigenvalues of measure differential equations. Proc. Am. Math. Soc. 143, 1991-2002 (2015)

10. Meng, G., Shen, K., Yan, P., Zhang, M.: Strong continuity of the Lidstone eigenvalues of the beam equation in potentials. Oper. Matrices 8, 889-899 (2014)

11. Meng, G., Yan, P., Zhang, M.: Minimization of eigenvalues of one-dimensional p-Laplacian with integrable potentials. J. Optim. Theory Appl. 156, 294-319 (2013)

12. Meng, G., Zhang, M.: Dependence of solutions and eigenvalues of measure differential equations on measures. J. Differ. Equ. 254, 2196-2232 (2013)

13. Mingarelli, A.B.: Volterra-Stieltjes Integral Equations and Generalized Ordinary Differential Expressions. Lecture Notes Math., vol. 989. Springer, New York (1983)

14. Savoye, P.: Equimeasurable rearrangements of functions and fourth order boundary value problems. Rocky Mt. J. Math. 26, 281-293 (1996)

15. Schwabik, Š.: Generalized Ordinary Differential Equations. World Scientific, Singapore (1992)

16. Trrdý, M.: Differential and integral equations in the space of regulated functions. Mem. Differ. Equ. Math. Phys. 25 1-104 (2002)

17. Wei, Q., Meng, G., Zhang, M.: Extremal values of eigenvalues of Sturm-Liouville operators with potentials in $L^{1}$ balls. J. Differ. Equ. 247, 364-400 (2009)

18. Zhang, M.: Extremal eigenvalues of measure differential equations with fixed variation. Sci. China Math. 53, 2573-2588 (2010) 